\title{
Elevated Concentration of Soluble Vascular Endothelial Cadherin Is Associated With Coronary Atherosclerosis
}

\author{
Takeshi Soeki, MD; Yoshiyuki Tamura, MD; Hisanori Shinohara, MD; \\ Koichi Sakabe, MD; Yukiko Onose, MD; Nobuo Fukuda, MD
}

\begin{abstract}
Background Vascular endothelial (VE)-cadherin, a $\mathrm{Ca}^{2+}$-dependent cell adhesion molecule, is expressed in atherosclerotic lesions by endothelial cells and is associated with neovascularization, although the relationship between circulating VE-cadherin and coronary artery disease has not been studied.

Methods and Results The plasma concentration of VE-cadherin was measured in peripheral blood (femoral artery) and the coronary sinus of 24 patients with acute myocardial infarction (AMI), 26 with stable angina pectoris (AP), 18 with old myocardial infarction (OMI), and 30 control subjects (Control) who had no coronary artery stenosis on angiography. For the patients with AMI, blood samples were obtained in the acute (day 1) and chronic (day 21) phases. The plasma concentration of VE-cadherin was measured by enzyme-linked immunosorbent assay. The correlation between the plasma VE-cadherin concentration and the Gensini score was also determined as an index of the severity of coronary atherosclerosis. The plasma concentrations of VE-cadherin (ng/ml) in both the peripheral and coronary sinus blood were higher in patients with AMI, AP, and OMI than in the control subjects, and were similar in the 3 groups with coronary artery disease (femoral artery: AMI 5.1 \pm 2.5 , AP 4.7 \pm 2.4 , OMI 4.5 \pm 3.3 , Control 2.6 \pm 2.3 ; coronary sinus: AMI 5.6 \pm 2.6 , AP 5.0 \pm 2.3 , OMI 5.0 \pm 2.9 , Control 2.4 \pm 2.1 , respectively). Plasma VE-cadherin concentrations were higher in the coronary sinus than peripheral blood samples in patients with AMI $(\mathrm{p}<0.01)$, AP $(\mathrm{p}<0.01)$, and OMI $(\mathrm{p}<0.05)$. The plasma VE-cadherin concentration was the same in the acute and chronic phases in patients with AMI. In the 3 groups of patients with coronary disease, both the peripheral plasma VE-cadherin concentration and the coronary sinus concentration correlated with the Gensini score $(r=0.32, p<0.01$ and $r=0.42, p<0.001$, respectively). Multiple regression analysis revealed that the plasma VE-cadherin concentration predicted the Gensini score independently of sex, age, hypertension, diabetes mellitus, smoking, and the lipid profiles.
\end{abstract}

Conclusion Increased secretion of VE-cadherin from the epicardial arteries is associated with the degree of coronary atherosclerosis, indicating the presence of atherosclerosis rather than disease activity. (Circ J 2004; 68: $1-5)$

Key Words: Atherosclerosis; Coronary artery disease; Vascular endothelial cadherin

$\mathbf{L}$ eukocyte binding to cellular adhesion molecules expressed on the surface of vascular endothelial cells appears to be one of the earliest events in the atherosclerotic process; ${ }^{1-3}$ pathologic studies have demonstrated focal expression of cellular adhesion molecules in human atherosclerotic plaques.,5 The extracellular portion of these adhesion proteins can be cleaved by proteolytic enzymes to form circulating molecules that can be detected in serum and are referred to as soluble cell adhesion molecules 6,7 Although the pathogenic role of these circulating adhesion molecules remains uncertain, recent cross-sectional studies have demonstrated that the concentrations of soluble adhesion molecules, including intercellular adhesion molecule-1 and vascular cell adhesion molecule-1, correlated with the extent of underlying atherosclerosis ${ }^{8-10}$ Furthermore, prospective cohort studies have indicated that elevated concentrations of some soluble adhesion molecules are associated with future cardiovascular events, both

(Received July 15, 2003; revised manuscript received October 2, 2003; accepted October 7, 2003)

Department of Cardiology and Clinical Research, National Zentsuji Hospital, Kagawa, Japan

Mailing address: Takeshi Soeki, MD, Department of Biochemistry, National Cardiovascular Center, 5-7-1 Fujishiro-dai, Suita, Osaka 565-8565, Japan..E-mail: tsoeki@ri.ncvc.go.jp in apparently healthy individuals ${ }^{11,12}$ and in patients with coronary artery disease $(\mathrm{CAD}) !^{13}$

Although the adhesion molecules responsible for heterotypical cell-to-cell interactions have received considerable attention, cellular adhesion molecules determining exclusively homotypical cell interactions in human atherosclerosis have not! ${ }^{14,15}$ Vascular endothelial (VE)-cadherin is a $\mathrm{Ca}^{2+}$-dependent cell adhesion molecule that associates with thea - and $\beta$-catenins to form a complex at endothelial cellto-cell contacts and is responsible for the organization of inter-endothelial junctions ${ }^{16,17}$ It was recently demonstrated that VE-cadherin is expressed in atherosclerotic lesions by endothelial cells and is associated with neovascularization, 15 but there has not been an examination of the relationship between the circulating VE-cadherin concentration and CAD. Therefore, our aim in this study was to determine whether the plasma soluble VE-cadherin concentration is associated with coronary atherosclerosis.

\section{Methods}

\section{Patients}

We studied 24 patients with acute myocardial infarction (AMI) (mean age: 67 years) who were hospitalized in the coronary care unit within $6 \mathrm{~h}$ of the onset of symptoms and 
Table 1 Demographics and Clinical Characteristics of the 4 Study Groups

\begin{tabular}{|c|c|c|c|c|}
\hline & $A M I$ & $A P$ & $O M I$ & Control \\
\hline$n$ & 24 & 26 & 18 & 30 \\
\hline$M / F$ & $19 / 5$ & $21 / 5$ & $10 / 8$ & $19 / 11$ \\
\hline Age (years) & $67 \pm 11$ & $65 \pm 7$ & $68 \pm 8$ & $65 \pm 10$ \\
\hline Smoking (n) & $11(45.8 \%)$ & $10(38.5 \%)$ & $8(44.4 \%)$ & $9(30.0 \%)$ \\
\hline Hypertension (n) & $10(41.7 \%)$ & $10(38.5 \%)$ & $8(44.4 \%)$ & $8(26.7 \%)$ \\
\hline Diabetes mellitus ( $n$ ) & $8(33.3 \%)$ & $7(26.9 \%)$ & $6(33.3 \%)$ & $5(16.7 \%)$ \\
\hline \multirow[t]{2}{*}{ Total cholesterol $(\mathrm{mg} / \mathrm{dl})$} & $202 \pm 40$ & $205 \pm 50$ & $198 \pm 44$ & $190 \pm 36$ \\
\hline & & L & & \\
\hline HDL-cholesterol $(\mathrm{mg} / \mathrm{dl})$ & $41 \pm 7$ & $47 \pm 9$ & $46 \pm 8$ & $51 \pm 9$ \\
\hline Triglycerides $(\mathrm{mg} / \mathrm{dl})$ & $122 \pm 62$ & $136 \pm 58$ & $121 \pm 63$ & $114 \pm 58$ \\
\hline
\end{tabular}

AMI, acute myocardial infarction; $A P$, angina pectoris; OMI, old myocardial infarction; $H D L$, high-density lipoprotein.

${ }^{*} p<<0.01 ; * p<0.05$.

who underwent successful coronary angioplasty, 26 patients with stable angina pectoris (AP) (mean age: 65 years) who had angiographic evidence of $\geq 90 \%$ coronary stenosis and had reversible perfusion defects on adenosine triphosphate stress thallium-201 myocardial scintigraphy, and 18 patients with old myocardial infarction (OMI) (mean age: 68 years) who had angiographic evidence of $\geq 90 \%$ coronary stenosis and no reversible perfusion defects on stress myocardial scintigraphy. The control group consisted of 30 subjects (mean age: 65 years) with chest pain, but no significant coronary stenosis on angiography $(<50 \%$ of luminal diameter).

AMI was diagnosed based on typical chest pain lasting $>30$ min with $>0.2 \mathrm{mV}$ of ST-T segment elevation in at least 2 contiguous leads on a standard 12-lead electrocardiogram and an increase in serum creatine kinase concentration that was at least twice the upper normal limit. AP was defined as typical anginal chest pain lasting between 10 and $30 \mathrm{~min}$ with $\geq 1 \mathrm{~mm}$ transient ST-segment depression or elevation $0.08 \mathrm{~s}$ after the $\mathrm{J}$ point, and no episodes of angina at rest or increasing angina. The serum creatine kinase activity had to be less than twice the upper limit of normal. Patients with OMI were enrolled in the study at least 6 months after the occurrence of AMI. Patients with significant concomitant diseases, such as malignancy or autoimmune disease, were excluded.

All patients gave written informed consent prior to participation in the study, which was approved by the institutional Committee on Human Research.

\section{Coronary Angiography}

All patients with AP and OMI underwent coronary angiography within 2 weeks of adenosine triphosphate stress thallium-201 myocardial scintigraphy. Coronary angiography was performed using the standard Judkins technique. Patients with coronary artery stenoses that were $\geq 90 \%$ of the vessel diameter in 1 or more epicardial vessels were enrolled in this study. The results were interpreted by 2 senior angiographers who had no knowledge of the results of the thallium imaging. We calculated the Gensini score $^{18}$ as the index of the severity of coronary atherosclerosis, based on the results of coronary angiography to examine the correlation between plasma concentration of VE-cadherin and Gensini score. Patients in the CAD subgroups (AMI, AP, and OMI) were divided into 4 groups based on the angiographically determined development of collaterals using the system designed by Rentrop et al ${ }^{19}$ (collateral grade 0-4) to evaluate the relationship between plasma VE-cadherin concentration and collateral grade.

\section{Blood Samples}

Blood samples for the measurement of VE-cadherin were obtained from both the peripheral circulation (femoral artery) and the coronary sinus, using a coronary sinus catheter (6F; Goodman, Nagoya, Japan), at the time of admission and again from the femoral artery 3 weeks after admission in patients with AMI. In the AP, OMI, and control groups, fasting blood samples were obtained from both the femoral artery and the coronary sinus just before coronary angiography. All blood samples were immediately centrifuged at $700 \mathrm{~g}$ for $10 \mathrm{~min}$, and the plasma samples were stored at $-70^{\circ} \mathrm{C}$ until the time of assay.

\section{Biochemical Analysis}

The plasma VE-cadherin concentration was measured by enzyme-linked immunosorbent assay (Bender MedSystems, Vienna, Austria) according to the manufacturer's instructions, with intra-assay and inter-assay coefficients of variation of $4.1 \%$ and $7.2 \%$. The sensitivity of this kit was $0.15 \mathrm{ng} / \mathrm{ml}$.

\section{Statistical Analysis}

All results are expressed as the mean \pm standard deviation. The differences in ratios were compared using the chi-square test. Because the distribution of the soluble VE-cadherin concentration was skewed, nonparametric analysis was performed for comparisons between groups (Kruskal-Wallis and Dunn tests) or between the acute and subacute phases of AMI (Wilcoxon signed-ranks test). The relationship between 2 factors was analyzed using the Pearson correlation coefficient. The correlation between the Gensini score and atherosclerotic risk factors was evaluated by multiple regression analysis. For multiple regression analysis, the following risk factors were regarded as covariates in addition to VE-cadherin: male sex, age, hypertension, diabetes mellitus, smoking, total cholesterol, high-density lipoprotein-cholesterol, and triglycerides. A value of $\mathrm{p}<0.05$ was considered statistically significant.

\section{Results}

\section{Patients Characteristics}

Table 1 summarizes the characteristics of each study group. The groups were comparable with respect to gender, age, and the concentrations of total cholesterol and triglycerides. The incidences of smoking, hypertension and dia- 

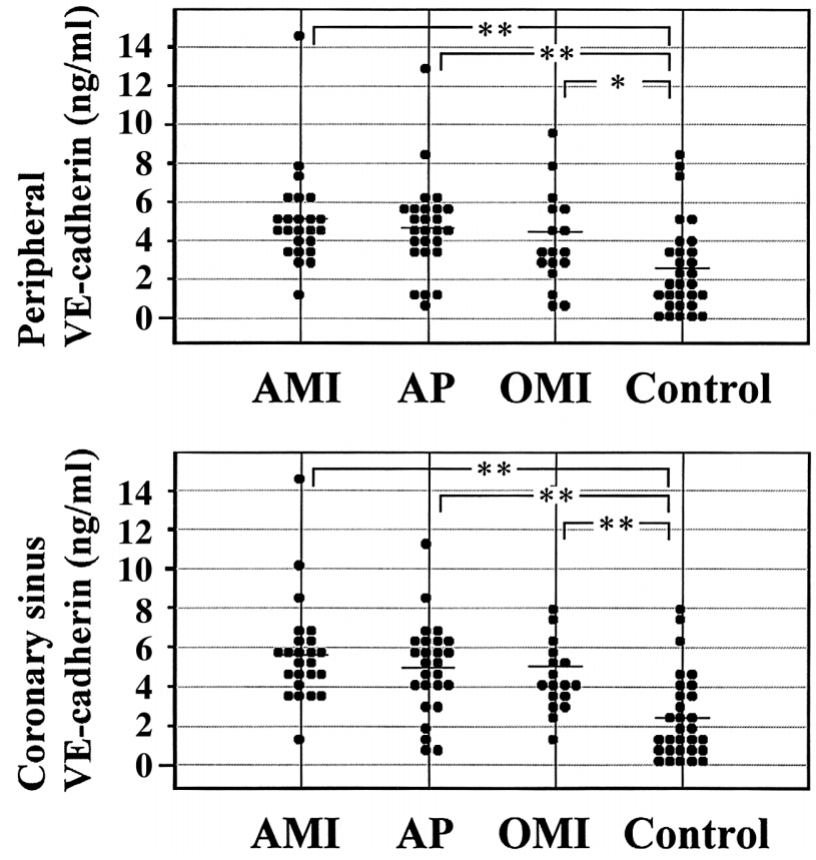

Fig 1. Plasma concentrations of vascular endothelial (VE)-cadherin in the peripheral and coronary sinus blood samples of control subjects and patients with AMI, AP, and OMI. Values are mean \pm SD. **p< 0.01 vs Control; $* \mathrm{p}<0.05$ vs control. AMI, acute myocardial infarction; AP, angina pectoris; OMI, old myocardial infarction.

betes mellitus were lower in controls, but the difference was not statistically significant. The high-density lipoprotein-cholesterol concentration was lower in the AMI group than in the other groups.

\section{Plasma VE-Cadherin Concentration}

The peripheral plasma concentration of VE-cadherin was significantly higher in patients with AMI, AP, and OMI than in the control subjects (Control), and did not differ between the 3 groups with CAD (AMI 5.1 \pm 2.5 , AP 4.7 \pm 2.4 , OMI 4.5 \pm 3.3 , and Control 2.6 $\pm 2.3 \mathrm{ng} / \mathrm{ml}$; Kruskal-Wallis test $\mathrm{p}<0.001$ ) (Fig 1, Upper). The plasma VE-cadherin concentration in the coronary sinus was also significantly higher in patients with AMI, AP, and OMI than in the control subjects and did not differ between the 3 groups with

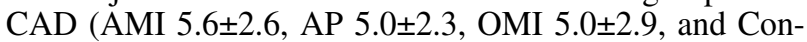
trol $2.4 \pm 2.1 \mathrm{ng} / \mathrm{ml}$; Kruskal-Wallis test $\mathrm{p}<0.0001$ ) (Fig 1, Lower). Plasma VE-cadherin concentrations were higher in the coronary sinus than peripheral blood samples in patients with AMI $(\mathrm{p}<0.01)$, AP $(\mathrm{p}<0.01)$, and OMI $(\mathrm{p}<0.05)$. In contrast, the plasma VE-cadherin concentration in the coronary sinus was similar to that in the peripheral blood sample in the control subjects $(p=0.55)$.

There were no significant differences in peripheral and coronary sinus plasma VE-cadherin concentrations between patients with and without hypertension, diabetes mellitus, and smoking (data not shown).

\section{Time-Course of the Plasma VE-Cadherin Concentration in Patients With AMI}

The peripheral plasma VE-cadherin concentration at the time of admission was similar to the concentration measured 3 weeks after admission in patients with AMI $(5.1 \pm 2.5$ vs $4.8 \pm 2.3 \mathrm{ng} / \mathrm{ml}, \mathrm{p}=0.80)$.
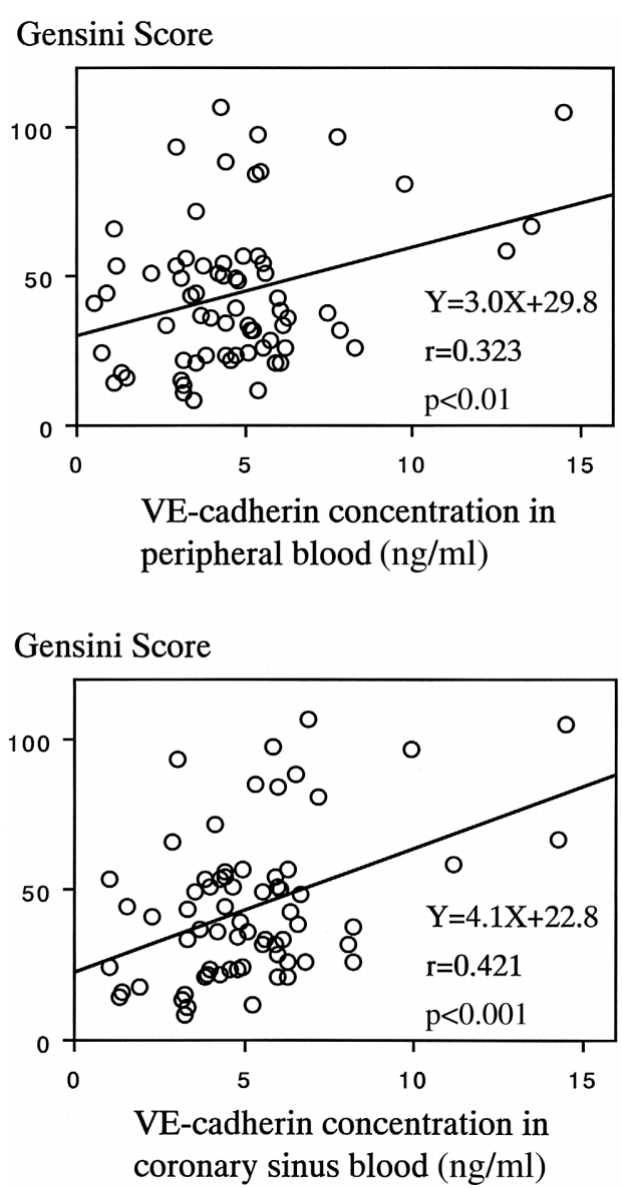

Fig 2. Correlation between the Gensini score and the plasma concentrations of vascular endothelial (VE)-cadherin in the peripheral and coronary sinus blood samples.

\section{Correlation Between Plasma VE-Cadherin Concentration and the Gensini Score}

In the AMI, AP, and OMI groups $(n=68)$, the peripheral plasma VE-cadherin concentration correlated with the Gensini score $(r=0.32, p<0.01)$, and the concentration in the coronary sinus correlated with the Gensini score more strongly ( $\mathrm{r}=0.42, \mathrm{p}<0.001)$ (Fig 2).

In all 4 groups, multiple regression analysis revealed that the plasma VE-cadherin concentration predicted the Gensini score independently of sex, age, hypertension, diabetes mellitus, smoking and the lipid profiles, including the serum concentrations of total cholesterol, high-density lipoprotein-cholesterol and triglycerides (Table 2). Diabetes mellitus and high-density lipoprotein-cholesterol concentrations also predicted the Gensini score independently, but their standard regression coefficients were lower than that of VE-cadherin.

\section{Correlation Between Plasma VE-Cadherin Concentration and the Degree of Collateral Development}

Neither peripheral nor coronary sinus plasma VEcadherin concentrations correlated with the collateral grade in the AMI, AP, and OMI groups (peripheral plasma VEcadherin, $\mathrm{r}=0.13, \mathrm{p}=0.30$; coronary sinus plasma VEcadherin, $\mathrm{r}=0.12, \mathrm{p}=0.35$ ). 
Table 2 Multiple Regression Analysis Between Gensini Score and Atherosclerotic Risk Factors

\begin{tabular}{lccc}
\hline \hline & Standard regression coefficient & t value & $p$ value \\
\hline VE-cadherin & 0.377 & 4.093 & $<0.0001$ \\
Male sex & 0.090 & 0.937 & 0.3511 \\
Age & -0.008 & -0.087 & 0.9311 \\
Hypertension & 0.082 & 0.885 & 0.3783 \\
Dabetes mellitus & 0.235 & 2.446 & 0.0164 \\
Smoking & 0.053 & 0.594 & 0.5540 \\
Total cholesterol & 0.186 & 1.776 & 0.0791 \\
HDL-cholesterol & -0.230 & -2.367 & 0.0201 \\
Triglycerides & -0.007 & -0.072 & 0.9428 \\
\hline
\end{tabular}

VE, vascular endothelial; HDL, high-density lipoprotein.

\section{Discussion}

The present study is the first to demonstrate that the soluble VE-cadherin concentration correlates with the extent of coronary atherosclerosis independently of other atherosclerotic risk factors including sex, age, hypertension, diabetes mellitus, smoking and the lipid profiles. Among the 'traditional' atherosclerotic risk factors, diabetes and high-density lipoprotein-cholesterol independently correlated with the extent of coronary atherosclerosis, but these factors were secondary to VE-cadherin in predicting the severity of coronary atherosclerosis. These results suggest that the plasma VE-cadherin concentration might be one of the most useful indicators of cardiovascular disease, such as atherosclerosis, independent of other risk factors.

In the present study, we also found that the plasma VEcadherin concentration in the coronary sinus had a stronger correlation with the Gensini score than that of the peripheral concentration. A recent histologic study demonstrated that VE-cadherin was expressed in atherosclerotic lesions by endothelial cells and was associated with some neovascularization, whereas in non-atherosclerotic segments of the vessel it was identified in the adventitia and the outer part of the media only 15 That finding, together with our clinical results, suggests that VE-cadherin plays an important role in atherosclerosis, and that coronary atherosclerotic lesions shed VE-cadherin into the coronary circulation.

We also found that plasma concentrations of VEcadherin in both the peripheral circulation and the coronary sinus were higher in patients with AMI, AP, or OMI than in the control subjects, and that the concentrations in the 3 groups of patients with CAD were similar. These results suggest that the plasma concentration is independent of coronary atherosclerotic plaque rupture. Recent studies have demonstrated that concentrations of soluble adhesion molecules for heterotypical cell-to-cell interactions, including intercellular adhesion molecule-1 and vascular cell adhesion molecule-1, correlate with both the extent of underlying atherosclerosis ${ }^{8-10}$ and cardiovascular events ${ }^{11-13}$ In addition, the concentrations of adhesion molecules, including P-selectin, have been reported to rise during the acute phase of AMI ${ }^{20-23}$ In contrast to these heterotypical adhesion molecules, the plasma concentration of VEcadherin, an adhesion molecule responsible for homotypical cell interactions, correlated with the degree of underlying coronary atherosclerosis and did not rise during the acute phase in patients with AMI in the present study. The cause of the differences in the dynamics between these soluble adhesion molecules in patients with acute coronary syndrome is unclear, but may reflect distinct roles for specific adhesion molecules related to heterotypic or homotypic interactions. Recent in vitro studies have showed that leukocyte adhesion induced disruption of the VE-cadherin complex and its loss from lateral junction localization, 24,25 suggesting that heterotypic adhesion molecules may trigger the downregulation of VE-cadherin in atherosclerotic plaque lesions.

Immunohistochemical studies have identified altered VE-cadherin expression in several tumors, including hemangiomas, ${ }^{26}$ glioblastomas, 27 and Kaposi's sarcoma, 28 which is not surprising because a series of shared molecular pathways, including the E-cadherin $\beta$-catenin pathway, have in common a significant role in the pathogenesis and progression of both atherosclerosis and cancer. ${ }^{29}$ However, the present study excluded patients with malignant disease to avoid confounding influences when assessing the direct relationship between VE-cadherin and coronary atherosclerosis.

Because a previous study showed that VE-cadherin in atherosclerotic lesions was associated with neovascularization, ${ }^{15}$ we examined the correlation between plasma VEcadherin concentration and the development of collateral vessels in patients with CAD. However, we could not find a correlation, which suggests that the circulating VEcadherin concentrations may not directly reflect the effects of local VE-cadherin on neovascularization. This study included a small number of patients, therefore the ability to generalize this correlation might be limited.

\section{Study Limitations}

First, we measured the plasma VE-cadherin concentration in a limited number of patients and at only a few time points. Second, we did not determine the concentrations of other adhesion molecules, such as intercellular adhesion molecule-1 or vascular cell adhesion molecule-1, simultaneously, so we could not clarify the relationships between homotypic and heterotypic adhesion molecules in patients with CAD. Third, we did not evaluate the relationship between soluble VE-cadherin concentration and 'systemic' atherosclerosis. To confirm the hypothesis that the peripheral VE-cadherin concentration reflects the extent of systemic rather than coronary atherosclerosis, the relation between peripheral VE-cadherin concentration and systemic atherosclerotic lesions, including those of the aorta, in which the earliest and most extensive atherosclerotic lesions usually occur ${ }^{30}$ need to be evaluated in further clinical studies.

\section{Conclusion}

We found that enhanced secretion of VE-cadherin from the arteries was associated with coronary atherosclerosis. 
Thus, the plasma VE-cadherin concentration might be a useful indicator of the presence of atherosclerosis, rather than of disease activity.

\section{References}

1. Cybulsky MI, Gimbrone MA Jr. Endothelial expression of a mononuclear leukocyte adhesion molecule during atherogenesis. Science 1991; 251: 788-791.

2. Li H, Cybulsky MI, Gimbrone MA Jr, Libby P. An atherogenic diet rapidly induces VCAM-1, a cytokine-regulatable mononuclear leukocyte adhesion molecule, in rabbit aortic endothelium. Arterioscler Thromb Vasc Biol 1993; 13: 197-204.

3. Fuster V, Corti R, Badimon JJ. The Mikamo Lecture 2002: Therapeutic targets for the treatment of atherothrombosis in the new millennium: Clinical frontiers in atherosclerosis research. Circ $J$ 2002; 66: 783-790.

4. Davies MJ, Gordon JL, Gearing AJ, Pigott R, Woolf N, Katz D, et al The expression of the adhesion molecules ICAM-1, VCAM-1, PECAM, and E-selectin in human atherosclerosis. J Pathol 1993; 171: $223-229$.

5. O'Brien KD, Allen MD, McDonald TO, Chait A, Harlan JM, Fishbein D, et al. Vascular cell adhesion molecule-1 is expressed in human coronary atherosclerotic plaques: Implications for the mode of progression of advanced coronary atherosclerosis. J Clin Invest 1993; 92: 945-951.

6. Rothlein R. A form of circulating ICAM-1 in human serum. J Immunol 1991; 147: 3788-3793.

7. Gearing AJH, Hemingway JK, Pigott R, Hughes J, Rees AJ, Cashman SJ. Soluble forms of vascular adhesion molecules E-selectin, ICAM1 and VCAM-1: Pathological significance. Ann NY Acad Sci 1992; 667: $324-331$.

8. Morisaki N, Saito I, Tamura K, Tashiro J, Masuda M, Kanzaki T, et al. New indices of ischemic heart disease and aging: Studies on the serum levels of soluble intercellular adhesion molecule-1 (ICAM-1) and soluble vascular sell adhesion molecule-1 (VCAM-1) in patients with hypercholesterolemia and ischemic heart disease. Atherosclerosis 1997; 131: $43-48$.

9. De Caterina R, Basta G, Lazzerini G, Dell'Omo G, Petrucci R, Morale M, et al. Soluble vascular cell adhesion molecule-1 as a biochemical correlate of atherosclerosis. Arterioscler Thromb Vasc Biol 1997; 17: 2646-2654.

10. Rohde LE, Lee RT, Rivero J, Jamacochian M, Arroyo LH, Briggs W, et al. Circulating cell adhesion molecules are correlated with ultrasound-based assessment of carotid atherosclerosis. Arterioscler Thromb Vasc Biol 1998; 18: 1765-1770.

11. Hwang SJ, Ballantyne CM, Sharrett AR, Smith LC, Davis CE, Gotto AM Jr, et al. Circulating adhesion molecules VCAM-1, ICAM-1, and E-selectin in carotid atherosclerosis and incident coronary heart disease cases: The Atherosclerosis Risk In Communities (ARIC) study. Circulation 1997; 96: 4219-4225.

12. Ridker PM, Hennekens CH, Roitman-Johnson B, Stampfer MJ, Allen J. Plasma concentration of soluble intercellular adhesion molecule 1 and risks of future myocardial infarction in apparently healthy men. Lancet 1998; 351: 88-92.

13. Blankenberg S, Rupprecht HJ, Bickel C, Peetz D, Hafner G, Tiret L, et al. Circulating cell adhesion molecules and death in patients with coronary artery disease. Circulation 2001; 104: 1336-1342.

14. Bobryshev YV, Lord RSA, Watanabe T, Ikezawa T. The cell adhesion molecule E-cadherin is widely expressed in human atheroscle- rotic lesions. Cardiovasc Res 1998; 40: 191-205.

15. Bobryshev YV, Cherian SM, Inder SJ, Lord RSA. Neovascular expression of VE-cadherin in human atherosclerotic arteries and its relation to intimal inflammation. Cardiovasc Res 1999; 43: $1003-$ 1017.

16. Lampugnani MG, Corada M, Caveda L, Breviario F, Ayalon O, Geiger B, et al. The molecular organization of endothelial cell to cell junctions: Differential association of plakoglobin, beta-catenin, and alpha-catenin with vascular endothelial cadherin (VE-cadherin). $J$ Cell Biol 1995; 129: 203-217.

17. Breviario F, Caveda L, Corada M, Martin-Padura I, Navarro P, Golay J, et al. Functional properties of human vascular endothelial cadherin (7B4/cadherin-5), an endothelium-specific cadherin. Arterioscler Thromb Vasc Biol 1995; 15: 1229-1239.

18. Gensini GG. A more meaningful scoring system for determining the severity of coronary heart disease. Am J Cardiol 1983; 51: 606-607.

19. Rentrop KP, Cohen M, Blanke H, Phillips RA. Changes in collateral channel filling immediately after controlled coronary artery occlusion by an angioplasty balloon in human subjects. J Am Coll Cardiol 1985; 5: $587-592$.

20. Kaikita K, Ogawa H, Yasue H, Sakamoto T, Miyao Y, Suefuji H, et al. Increased plasma soluble intercellular adhesion molecule-1 levels in patients with acute myocardial infarction. Jpn Circ J 1997; 61: $741-748$

21. Ikeda H, Nakayama H, Oda T, Kuwano K, Muraishi A, Sugi K, et al. Soluble form of P-selectin in patients with acute myocardial infarction. Coron Artery Dis 1994; 5: 515-518.

22. Shimomura H, Ogawa H, Arai H, Moriyama Y, Takazoe K, Hirai N, et al. Serial changes in plasma levels of soluble P-selectin in patients with acute myocardial infarction. Am J Cardiol 1998; 81: 397-400.

23. Mulvihill N, Foley B, Ghaisas N, Murphy R, Crean P, Walsh M. Early temporal expression of soluble cellular adhesion molecules in unstable angina and subendocardial myocardial infarction. Am J Cardiol 1999; 81: 1265-1267.

24. Allport JR, Ding H, Collins T, Gerritsen ME, Luscinskas FW. Endothelial-dependent mechanisms regulate leukocyte transmigration: A process involving the proteasome and disruption of the vascular endothelial-cadherin complex at endothelial cell-to-cell junctions. $J$ Exp Med 1997; 186: 517-527.

25. Del Maschio A, Zanetti A, Corada M, Rival Y, Ruco L, Lampugnani MG, et al. Polymorphonuclear leukocyte adhesion triggers the disorganization of endothelial cell-to-cell adherens junctions. J Cell Biol 1996; 135: 497-510.

26. Martin-Padura I, De Castellamau C, Uccini S, Pilozzi E, Natali PG, Nicotra MR, et al. Expression of VE (vascular endothelial)-cadherin and other endothelial-specific markers in haemangiomas. $J$ Patho 1995; 175: $51-57$

27. Vitolo D, Paradiso P, Uccini S, Ruco LP, Baroni CD. Expression of adhesion molecules and extracellular matrix proteins in glioblastomas: Relation to angiogenesis and spread. Histopathology 1996; 28: 521 528.

28. Uccini S, Ruco LP, Monardo F, Stoppacciaro A, Dejana E, La Parola IL, et al. Co-expression of endothelial cell and macrophage antigens in Kaposi's sarcoma cells. J Pathol 1994; 173: 23-31.

29. Ross JS, Stagliano NE, Donovan MJ, Breitbart RE, Ginsburg GS Atherosclerosis and cancer: Common molecular pathways of disease development and progression. Ann NY Acad Sci 2001; 947: $271-$ 293.

30. Neunteufl T, Maurer G. Noninvasive ultrasound techniques for the assessment of atherosclerosis in coronary artery disease. Circ J 2003; 67: $177-186$. 